\title{
Compressive Behavior of Hybrid Composites
}

\author{
Chandra S. Yerramalli and Anthony M. Waas*
}

\author{
Composite Structures Laboratory, \\ Department of Aerospace Engineering, \\ University of Michigan, Ann Arbor, MI 48109-2140
}

February 11, 2003

\begin{abstract}
The response of hybrid glass/carbon polymer matrix composites was studied under compression. Experiments were performed under both static and dynamic loading conditions. A overall fiber volume fraction of $30 \%$ was chosen for the composite. The ratio of glass fibers to carbon fibers were varied to get a range of hybrid ratios starting from pure glass to a mixture of glass and carbon and pure carbon. The composite specimens were cylindrical in shape, approximately $6.77 \mathrm{~mm}$ in diameter, with a gage length of approximately $12.5 \mathrm{~mm}$. Under static conditions, it was observed that the failure strength decreased as the hybrid ratio increased, with the minimum decrease coinciding with a ratio of $50 \%$ glass and $50 \%$ carbon. The elastic modulus was found to be well approximated by a rule of mixtures formulation. The failure mechanism was observed to change from splitting for glass dominated composites to kinking in carbon dominated composites. Dynamic experiments were performed on a Split Hopkinson Pressure Bar (SHPB) test setup. The SHPB allows for strain rates upto $2500 \mathrm{\epsilon} / \mathrm{s}$. The effect of strain rate on failure mode and failure strength was investigated.
\end{abstract}

\section{Introduction}

Fiber composites allow tailoring of properties according to requirements through a choice of the constituents (fiber and matrix) in an optimum manner. Hybridization of fiber composites enhances

${ }^{*}$ Postdoctoral Research Fellow and Professor of Aerospace Engineering, respectively, University of Michigan. Assoc. Fellow AIAA, Copyright (C) 2002 by Anthony M. Waas. Published by the AIAA, with permission. this property of fiber reinforced composite materials. Hybridization can be done in both architecture (e.g. plain weave woven fabrics with stitching in the transverse direction) or materials (by using two or more types of reinforcing fibers). Hybridization in composites can lead to benefits in cost and enhancement of mechanical properties. Previous studies on the mechanical behavior of hybrid composites have focussed mainly on tensile and impact behavior, Aveston and Sillwood [1976], Dorey et al. [1978]. An exception to this are the works of Piggott and Harris [1981], Yao and Chou [1989], and Martinez et al. [1981], who studied the static compressive response of hybrid glass/carbon composites. Investigations on the dynamic compressive stress response and strain rate effects on compressive strength of hybrid (glass/carbon) composites has not been reported in the open literature. Waas et al. [1997], Lee [1998], and Yuan and Takeda [2000] studied the compressive behavior of unidirectional glass and unidirectional carbon composites under high strain rates. Waas et al. [1997] and Yuan and Takeda [2000] conducted high strain rate tests on glass fiber composites at strain rates of $\dot{\epsilon}=1000 \mathrm{~s}^{-1}$. They observed the failure stress to increase with increasing strain rate. Tests were also conducted by varying the specimen temperature. It was observed that the failure strength of the specimen was a decreasing function of increasing temperature. This was due to the degradation of the matrix mechanical properties. The failure mechanism was observed to be splitting in case of glass fiber composites and kinking in the case of carbon fiber composites.

Results presented in the literature have shown that the failure strength and failure mechanism of fiber reinforced composites are influenced by a number of different parameters(Yerramalli and Waas [2001]). Thus, hybridization of composites by mixing carbon and glass fibers will lead to results 
that reinforce and delineate conclusions regarding the failure mechanism, failure strength prediction and the dependancy of failure mechanism on parameters such as fiber type, interfacial fracture energy and matrix mechanical properties. Based on the results presented by Fleck [1997], Lee and Waas [1999], Vogler and Kyriakides [1999], and Vogler et al. [2000], it is known that the compression strength of polymer composites depends on the fiber type, matrix shear properties, fiber/matrix interface toughness and misalignment of the fibers. In case of pure carbon composites, kinking is the dominant failure mechanism whereas for glass composites, splitting is found to be the dominant failure mechanism.

In their paper, Piggott and Harris [1981] studied the effect of hybridization of glass/carbon composites experimentally. They also conducted experiments on hybrid(carbon/kevlar) composites under pure compression. They observed that the effect of hybridization(glass/carbon) was to reduce the compressive strength of the composite. However, in the case of carbon/kevlar hybridization, they observed an increase in strength with hybridization. In the prese, results obtained from studying glass/carbon fiber hybrid polymer matrix composites under static and dynamic compressive loading are presented. For this purpose, an overall fiber volume fraction, $V_{f}=0.3$ was chosen. The fiber content of individual constituents were varied from pure glass to pure carbon, while keeping the overall fiber volume fraction fixed at 0.3. Results presented in Lee and Waas [1999], showed that the failure mode underwent a transition from pure splitting to splitting/kinking around $V_{f} \approx 0.3$. Thus, it was felt that an overall $V_{f} \approx 0.3$ was ideal for studying the influence of different constituents on the failue mechanisms.

\section{Experiments}

Pure compression tests were performed on cylindrical composite specimens. The specimen diameter was approximately $6.8 \mathrm{~mm}$ and the gage length of the specimen was $12.7 \mathrm{~mm}$. Two back to back strain gages were attached to record the strain in the gage section and also to check the loading alignment. The specimens were manufactured with a range of hybrid ratios of glass/carbon. The specimens were compressed in a MTS hydraulically actuated machine for static loading and in a split Hopkinson pressure bar (SHPB) setup for dynamic loading. Details of the specimen manufacturing process and the SHPB setup are included in Yerramalli [2003]. The total volume fraction of fibers, $V_{f}$, was kept constant at 0.3 . The ratio of glass to carbon fibers was varied from the case of pure glass, $V_{g c}=1$ to pure carbon, $V_{c c}=1$, with the following constraint; $V_{g c}+V_{c c}=1$. The individual fiber volume fraction at any ratio is obtained by the following relations for glass, $V_{f g}=r_{0 g}^{2} / r_{c}^{2}$ and for carbon, $V_{f c}=r_{0 c}^{2} / r_{c}^{2}$. Here, $r_{0 c}$ and $r_{0 g}$ are the carbon fiber and glass fiber radius, respectively. $r_{c}$ is the composite cylinder radius. In the experiments (for both static and dynamic), the values of $V_{g c}$ chosen were $\{1,0.9,0.7,0.5,0.3,0.1,0\}$ and the value for $V_{c c}$ was obtained from the constraint relation. These new terms for the individual fiber volume fractions are defined below in terms of the fiber radius.

$$
\begin{aligned}
V_{g} & =\frac{r_{0 g}^{2}}{r_{m g}^{2}} \\
V_{c} & =\frac{r_{0 c}^{2}}{r_{m c}^{2}} \\
V_{g c} & =\frac{r_{m g}^{2}}{r_{c}^{2}} \\
V_{c c} & =\frac{r_{m c}^{2}}{r_{c}^{2}} \\
V_{f g} & =V_{g c} \times V g \\
V_{f c} & =V_{c c} \times V c
\end{aligned}
$$

\subsection{Static Compression}

The hybrid composites were tested to failure in a quasi-static manner. Typical stress vs strain responses obtained from the experiments during the testing of hybrid composites are plotted in figure 1. It can be observed that as the carbon fiber volume fraction, $V_{f c}$, increases, the failure strain decreases. The minimum strain to failure does not correspond to that of pure carbon but to a hybrid ratio with a very low percentage of glass fibers. The static results on hybrid composites show that the effect of mixing carbon to glass fibers is to reduce the compressive strength as shown in figure 2. The failure mechanism changed from splitting in pure glass composites $\left(V_{g c}=1\right)$ to kinking failure in carbon composites $\left(V_{c c}=1\right)$ as the percentage of carbon fibers increased in the hybrid composite. The change in failure mechanism was found to occur around a hybrid ratio of 0.5 . The measured elastic modulus of the hybrid composite was plotted as a function of the hybrid ratio and, analytically, the composite rule of mixtures was found to adequately approximate the experimental data as can be seen in figure 3. Similarly, it was observed that as the percent- 
age of carbon fibers increased in the composite, the strain to failure decreased. Thus, the use of hybrid glass/carbon composites did not lead to an increase in static compressive strength.

\subsection{Dynamic Compression}

The hybrid composite specimens were also studied under high strain rate loading using a split Hopkinson pressure bar (SHPB) test setup. Details of the UM Aerospace department SHPB and the technique of establishing stress-strain data and strength data under dynamic loading are given in Yerramalli [2003].

The SHPB setup consists of an impactor bar, incident bar and a transmission bar. Usually a fourth bar called the throw-off bar acts like a momentum trap. In the present SHPB setup, the four bars were made of $440 \mathrm{C}$ case hardened stainless steel. The specimen is sandwiched between the incident bar and the transmission bar. The specimen/bar interfaces are greased so as to reduce friction. The specimen length and diameter are kept very small compared to the length of the SHPB bars. This ensures that the one dimensional wave propagation assumptions are met and also that the specimen attains a uniform state of strain in a short time as compared to the duration of the loading pulse. The SHPB setup was first used to test aluminum6060 alloy specimens to validate the experimental setup and the computer programs that are subsequently used to extract the specimen stress and strain data from the strain gage signals. This particular alloy of aluminum is strain rate independent and can be useful in validating the SHPB test setup. As shown in figure 4 and figure 5 the compressive stress response of both specimens under varying strain rates is approximately same. Once the setup and the computer programs were validated, the hybrid composite specimens were examined under high strain rate loading conditions. Figure 6 shows the variation of stress with strain rate. It can be seen that initially the strain rate is varying and it is only in a short period before failure that the strain rate achieves a nearly constant value. In case of high strain rate tests on hybrid composites, the failure strength was observed to increase at each hybrid ratio (glass/carbon) as shown in figure 7 . There is wide scatter in the data due to which definite conclusions on the dependancy of failure strength on strain rate cannot be drawn. However, in general, it can be seen that the failure strength increases with increasing strain rate for all hybrid ratios. Typical stress response curves obtained for a composite with hybrid ratio of $V_{g c}=0.5$ is shown in figure 8. A typical oscilloscope trace of the strain gage signals obtained from the strain gages on the incident bar and the transmission bar are shown in figure 9. The specimens used in the high strain rate tests were completely destroyed. The hybrid composites with greater percentage of glass fibers exhibited brooming type of failure surfaces. In case of hybrid composites with higher percentage of carbon fibers, the failure surfaces did not show brooming. For all hybrid ratios, glass fiber composites show characteristics of splitting failure like brooming and disintegration of matrix. The exact failure mechanism could not be established in these tests since the specimen undergoes repeated loading under a series of compressive stress pulses. This leads to complete disintegration of the specimens.

\section{Analysis}

A model similar in concept to the concentric cylinder model is utilized for analysing the hybrid composites. The hybrid composite is assumed to consist of an assemblage of space filling concentric cylinders of carbon/matrix and glass/matrix distributed uniformly and mixed according to their respective constituent volume fractions and with an overall fiber volume fraction, $V_{f}=0.3$, as shown in figure 7. Thus, for example, for a hybrid ratio of 0.3 , ie. $V_{g c}=0.3$, the glass cylinder radius will be $r_{m g}=\left(V_{g c} / V_{f g}\right) \times r_{o g}^{2}$ and the carbon cylinder radius will be $r_{m c}=\left(V_{c c} / V_{f c}\right) \times r_{o c}^{2}$. In the experiments, a failure mode transition was observed as the percentage of glass fibers reduced from $100 \%$ to $0 \%$ by the replacement of glass fibers by carbon fibers. Hence, both kinking and splitting failure mechanisms are modeled at the individual concentric cylinder level and next assembled to obtain the composite behavior.

\subsection{Splitting and Kinking}

In case of splitting analysis, the expressions for stresses, strains and the expression for compliance remain the same as those of the splitting model presented in Yerramalli and Waas [2002a]. The only difference being that these expressions are now calculated for the individual components of the hybrid composites. Thus, in case of a glass/carbon hybrid composite, the expressions for critical compressive stress (from Yerramalli and Waas [2002a])) can be 
written as follows.

$$
\begin{aligned}
\sigma_{g}^{s p} & =\sqrt{\frac{8 V_{f}^{2} \gamma_{f g}}{r_{0 g}\left(1 / \delta_{g}-\beta_{g}\right)}} \\
\sigma_{c}^{s p} & =\sqrt{\frac{8 V_{f}^{2} \gamma_{f c}}{r_{0 c}\left(1 / \delta_{c}-\beta_{c}\right)}}
\end{aligned}
$$

Here, the subscripts $g$ and $c$ stand for glass and carbon respectively and the superscripts $s p$ and $k$ are for splitting and kinking failure mechanisms. Similarly, for kinking, the critical kinking stresses for both glass and carbon composite cylinder, from Yerramalli and Waas [2002b], are

$$
\begin{aligned}
\sigma_{g}^{k} & =\frac{\frac{1}{\gamma_{r m g}^{3}} \int_{0}^{\gamma_{r m g}} \tau_{g}(\gamma) \gamma^{2} d \gamma}{\frac{\phi_{g}}{3}+\frac{\gamma_{r m g}}{4}} \\
\sigma_{c}^{k} & =\frac{\frac{1}{\gamma_{r m c}^{3}} \int_{0}^{\gamma_{r m c}} \tau_{c}(\gamma) \gamma^{2} d \gamma}{\frac{\phi_{c}}{3}+\frac{\gamma_{r m c}}{4}}
\end{aligned}
$$

In the above equations, (2)-(4), $\gamma_{f}$ is the fracture energy, $\delta$ and $\beta$ are related to the elastic constants of the constituents, $\tau(\gamma)$ is the shear response of the composite (either glass or carbon based on the subscript). $\gamma_{r m g}$ and $\gamma_{r m c}$ are the shear strains at the glass and carbon composite cylinder outer radius, respectively, as indicated in figure 10. $\phi$ is the fiber misalignment angle with a subscript denoting glass or carbon as the case may be.

First, an iso-strain assumption to assemble the individual concentric cylinder level results and extract composite level properties is adopted. To ensure compatibility between the glass concentric cylinder and the carbon concentric cylinder, the axial strains in both composite cylinders are equated. From, the 3D stress analysis results in Yerramalli and Waas [2002a], expressions for strains are obtained. Then,

$$
\begin{aligned}
\epsilon_{f g}^{3 D} & =\epsilon_{f c}^{3 D} \\
\frac{2 \beta^{g} P_{g}}{\pi r_{o g}^{2}} & =\frac{2 \beta^{c} P_{c}}{\pi r_{o c}^{2}} \\
P_{c}=P_{g} \frac{\beta^{g}}{\beta^{c}} \frac{r_{o c}^{2}}{r_{o g}^{2}} &
\end{aligned}
$$

Using the expressions for fiber volume fractions given in equation (1), equation (6) can be written as

$$
P_{c}=P_{g} \frac{\beta^{g}}{\beta^{c}} \frac{V_{c c}}{V_{g c}}
$$

Equation (7), gives a relation between the load carried by a carbon composite cylinder, $P_{c}$, in terms of the load carried by a glass composite cylinder, $P_{g}$, at a given hybrid ratio as determined by $V_{c c}$ and $V_{g c}$. To obtain an expression for the total load carried by the complete composite, we need to rely on stress equilibrium along the axial direction to obtain a relation between the loads carried by individual constituents and the total load, $P_{\text {total }}$. Thus,

$$
\begin{aligned}
P_{\text {total }} & =P_{c}+P_{g} \\
& =P_{g}\left[1+\left(\beta^{g} / \beta^{c}\right)\left(V_{c c} / V_{g c}\right)\right]
\end{aligned}
$$

Equation (8), provides an expression to calculate the total load carried by the hybrid composite when a critical condition is reached for any of the individual constituent composite cylinders (either splitting or kinking). Defining, $\left(\beta^{g} / \beta^{c}\right)\left(V_{c c} / V_{g c}\right)=\psi$, we can write

$$
\begin{aligned}
& P_{g}=\frac{P_{t o t a l}}{(1+\psi)} \\
& P_{c}=\frac{P_{t o t a l} \psi}{(1+\psi)}
\end{aligned}
$$

The stress in a glass concentric cylinder can be obtained by referring to figure 10 and noting that $r_{m g}$ is the radius of the glass composite cylinder. Then, using equations (9), it follows that

$$
\begin{aligned}
\sigma_{g} & =\frac{P_{g}}{\pi r_{m g}^{2}} \\
& \text { but } \pi r_{m g}^{2}=V_{g c} \pi r_{c}^{2} \\
& =\frac{P_{t o t a l}}{(1+\psi) \pi r_{c}^{2} V_{g c}} \\
& =\frac{\sigma_{\text {total }}}{(1+\psi) V_{g c}}
\end{aligned}
$$

Therefore, the total stress expression can be written as

$$
\sigma_{\text {total }}=\sigma_{g}(1+\psi) V_{g c}
$$

Similarly, the total stress, $\sigma_{\text {total }}$, in terms of the stress in carbon concentric cylinder, $\sigma_{c}$, is obtained by following the above procedure. This results in,

$$
\sigma_{\text {total }}=\sigma_{c}(1+1 / \psi) V_{c c}
$$

In equation (12) and equation (13), $\sigma_{g}$ and $\sigma_{c}$ are the values calculated by equations (2-4). This completes the iso-strain failure model in the hybrid composite.

Next, an iso-stress assumption to model failure is considered. Previous work on compressive failure by Yao and Chou [1989] and Martinez et al. [1981], showed that unidirectional hybrid composites display a wide disparity in strain between the glass 
and carbon fibers. That is, when a hybrid composite containing a mixture of a flexible fiber and relatively stiff fiber are loaded in compression, the stiffer fiber reaches a critical stress state earlier than the compliant fiber. Thus, an iso-strain assumption will lead to a prediction of increase in strength for the case of addition of stiffer fibers (carbon) to a less stiff fiber (glass) reinforced composite. However, in the complementary iso-stress approach, it is assumed that the composite carries a stress, $\sigma$ which is transmitted to both glass concentric cylinder and a carbon concentric cylinder as schematically shown in figure 10. Thus, the critical composite stress is calculated when either of the constituent cylinders first reaches a critical stress state. For instance, if the glass concentric cylinder reaches the condition of splitting, then the critical failure stress is calculated in glass composite using equation (2). As the volume fraction of glass decreases, the compressive strength will now decrease.

In essence, the iso-strain approach and isostress approach provide bounds for the predictions of hybrid composite compressive strength. However, previous experimental results reported in Martinez et al. [1981] and the present experimental results suggest that an iso-stress approach is appropriate for the study of hybrid composites that contain fibers of disparate axial stiffness, and are dominated by the more compliant fiber. Results as predicted by the iso-strain model (carbon kinking) and the isostress model (glass splitting) are shown in figure 11, where, experimental results are also presented. It can be seen that, for small values of $V_{g c}$, the isostrain kinking model captures the experimental data very well. Beyond $V_{g c}=0.15$, the iso-strain kinking model underestimates the compressive strength. This is because, as $V_{g c}$ increases, the expressions for kinking (equation (4) based on the assumptions of pure kinking ceases to be valid. In a hybrid composite, the surrounding glass fibers offer more constraint to kinking (confinement) and thus, the kinking expression of equation (4) will underestimate the compressive strength. On the other hand, in this region, $0.5<V_{g c}<1$, the iso-stress model adequately capture the experimental data for compressive strength. Thus, when the compliant fiber dominates the hybrid composite, the iso-stress model predictions capture the experimental data, while for hybrid composites dominated by the stiffer fiber, the iso-strain model appears to capture the experimental strength data. It is to be noted that both models must be used with the appropriate failure mechanism as observed in the experiment. A mechanism based fail- ure modeling approach as presented here appear to adequately explain the static compressive strength data for hybrid composites.

\section{Conclusions}

The reported experimental results on glass/carbon hybrid composites suggest that, mixing of glass and carbon fibers in a single composite leads to a deterioration in the measured strength of the composite. The splitting and kinking models of failure for pure composites were modified to account for the presence of another type of fiber in the composite. Using equation (12) and equation (13), the total stress on the composite when either of the individual composite cylinder reaches the critical stress in splitting or kinking was obtained. The total stress values obtained by using glass and carbon composite cylinder stresses are plotted in figure 11. Here, the total stress predicted by the splitting of glass is consistently increasing with the addition of carbon fibers. This is due to the fact that the addition of carbon fibers increases the value of $\psi$, which leads to an increase in the total stress, $\sigma_{\text {total }}$ from equation (12). Also, the total stress predicted by the splitting of carbon composite cylinders consistently decreases with increasing glass fiber volume fraction or decreasing carbon fiber volume fraction. The total stress predicted by the iso-strain model (carbon kinking) for $\phi=3^{0}$ is seen to match with the experimental data upto $V_{f g}=0.15$. Beyond this glass volume fraction, the prediction provides a lower bound for the predicted values. However, it should be noted that the failure mechanism was not pure kinking beyond glass volume fraction of 0.15. Explaining the decrease in failure stress due to the addition of carbon fibers to a glass composite is not possible based on an iso-strain model. However, the decrease can be explained by using a iso-stress model as shown in figure 11 .

In case of high strain rate compressive strength of the hybrid composites, it can be seen that the failure strength does show a definite increase at each hybrid ratio but the scatter in data makes it difficult to find a specific relation between the strain rate and the strength. However, as can be seen from figure 12, the failure strength shows an increase across all hybrid ratios as compared to the static test data. 


\section{Acknowledgements}

We are grateful to the Army Research Office for financial support through ARO grant DAAG55-981-0317 with Dr. Bruce LaMattina as the scientific monitor.

\section{References}

J. Aveston and J. M. Sillwood. Synergistic fiber strengthening in hybrid composites. Journal of Materials Science, 11:1877-83, 1976.

G. Dorey, G. R. Sidey, and J. Hutchings. Impact properties of carbon fiber-kevlar49 fiber hybrid composites. Composites, 9:25-30, 1978.

M. R. Piggott and B. Harris. Compression strength of hybrid fibre-reinforced plastics. Journal of $M a-$ terials Science, 16:687-693, 1981.

Li Ning Yao and Tsu-Wei Chou. Analysis of hybrid effect in unidirectional composites under longitudinal compression. Composite Structures, 12: $27-37,1989$.

G. M. Martinez, M. R. Piggott, D. M. R. Bainbridge, and B. Harris. The compression strength of composites with kinked, misaligned and poorly adhering fibres. Journal of Materials Science, 16:28312836, 1981.

A. M. Waas, S. H. Lee, and N. Takeda. Dynamic compressive failure of glass/polyester composites. In Proceedings of American Society of Composites Meeting, November 1997.

S. H. Lee. Compressive behavior of fiber reinforced unidirectional composites. $\mathrm{PhD}$ thesis, Aerospace Engineering Department, University of Michigan, Ann Arbor, 1998.

J. Yuan and N. Takeda. Impact compressive failure of gfrp unidirectional composites. Science and Engineering of Composite Materials, 9(1):1-9, 2000.

Chandra S. Yerramalli and A. M. Waas. Failure of composites under combined loading. In Proceedings of 2001 ASME International Mechanical Engineering Congress and Exposition, New York, November 11-16 2001.

N. A. Fleck. Compressive failure of fiber composites. In Advances in applied mechanics, volume 33, pages 43-117. Academic Press, New York, 1997.
S. H. Lee and A. M. Waas. Compressive response and failure of fiber reinforced unidirectional composites. International Journal of Fracture, 100: 275-306, 1999.

T. J. Vogler and S. Kyriakides. Inelastic behavior of an as4/peek composite under combined transverse compression and shear. part I: experiments. International Journal of Plasticity, 15:783-806, 1999.

T. J. Vogler, S. Y. Hsu, and S. Kyriakides. Composite failure under combined compression and shear. International Journal of Solids and Structures, 37: 1765-1791, 2000.

Chandra Sekher. Yerramalli. A Mechanism Based Modeling Approach to Failure in Fiber Reinforced Composites. $\mathrm{PhD}$ thesis, Aerospace Engineering Department, University of Michigan, Ann Arbor, 2003.

Chandra S. Yerramalli and A. M. Waas. Compressive splitting failure of composites using modified shear lag theory. International Journal of Fracture, 115:27-40, May 2002a.

Chandra S. Yerramalli and A. M. Waas. A failure criterion for fiber reinforced polymer composites under combined compression-torsion loading. Accepted in International Journal of Solids and Structures, 2002b.

\begin{tabular}{|c|c|c|c|c|}
\hline$V_{f g}$ & \multicolumn{3}{|c|}{ Strength $(M P a)$} & Ave.Strength $(M P a)$ \\
\hline 0 & 425 & - & - & 425 \\
\hline 3 & 320 & 250 & 340 & 303.3 \\
\hline 9 & 385 & 345 & 320 & 350 \\
\hline 15 & 300 & 265 & 235 & 296.7 \\
\hline 21 & 345 & 340 & 320 & 335 \\
\hline 27 & 430 & 405 & 385 & 406.7 \\
\hline 30 & 484 & 520 & 480 & 494.7 \\
\hline
\end{tabular}

Table 1: Compressive strength of hybrid composites 


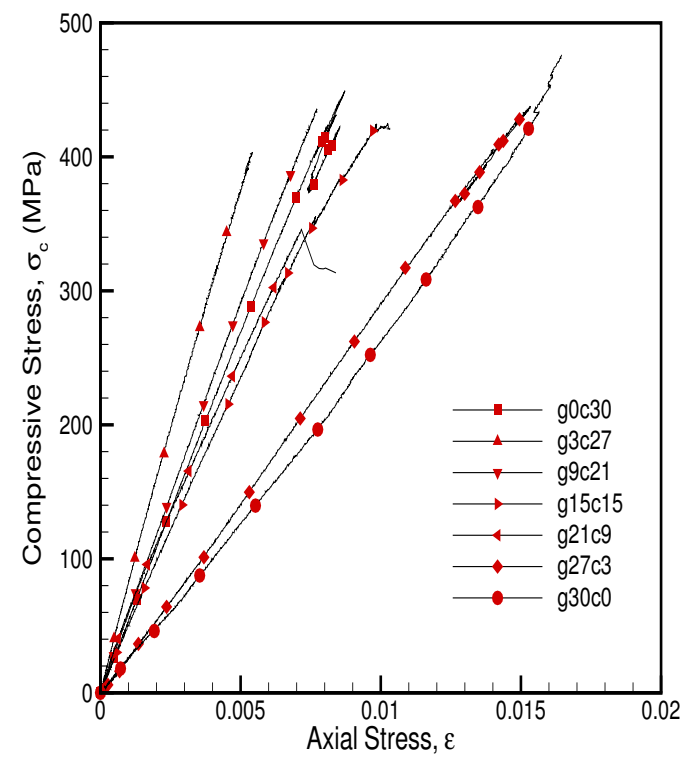

Figure 1: Typical compressive response of hybrid composites as a function of the hybrid ratio for a global fiber volume fraction of $30 \%$

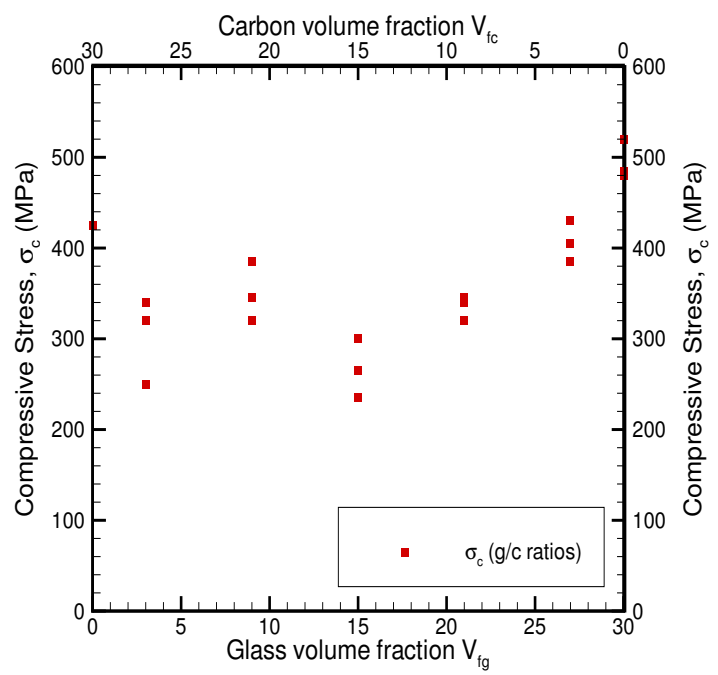

Figure 2: Variation of failure strength as a function of the hybrid ratio for a global fiber volume fraction of $30 \%$

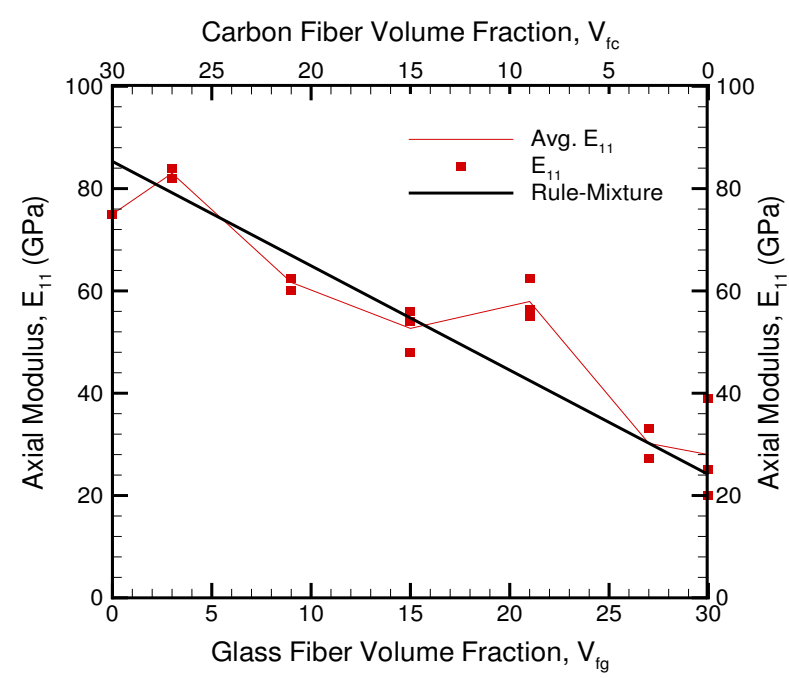

Figure 3: Variation of elastic modulus as a function of the hybrid ratio for a global fiber volume fraction of $30 \%$

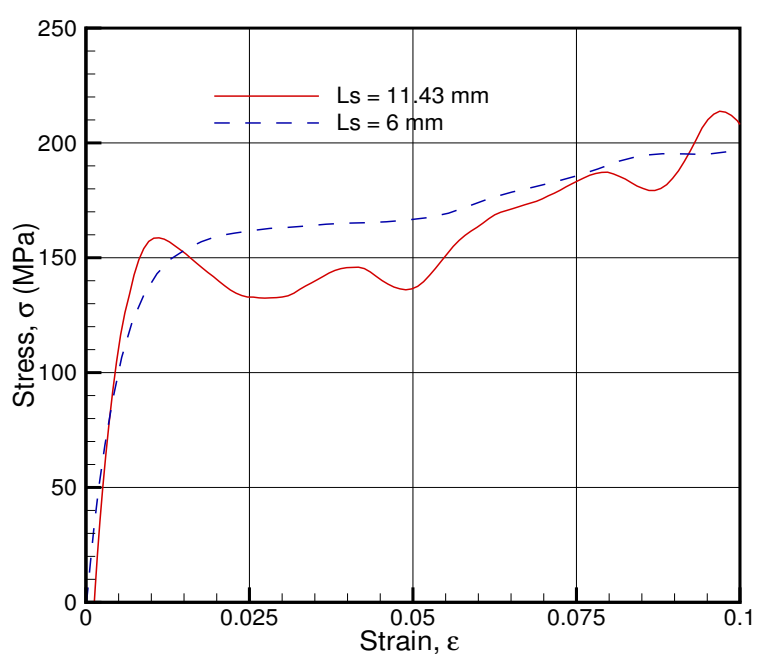

Figure 4: The compressive stress response of a Aluminum6060 alloy under high strain rate tests. 


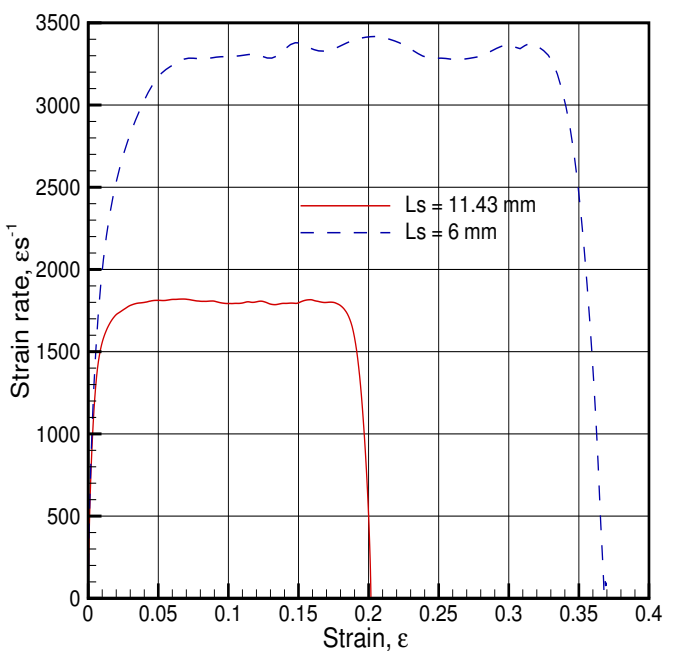

Figure 5: Variation of strain rate with the applied strain for aluminum6060 alloy specimens

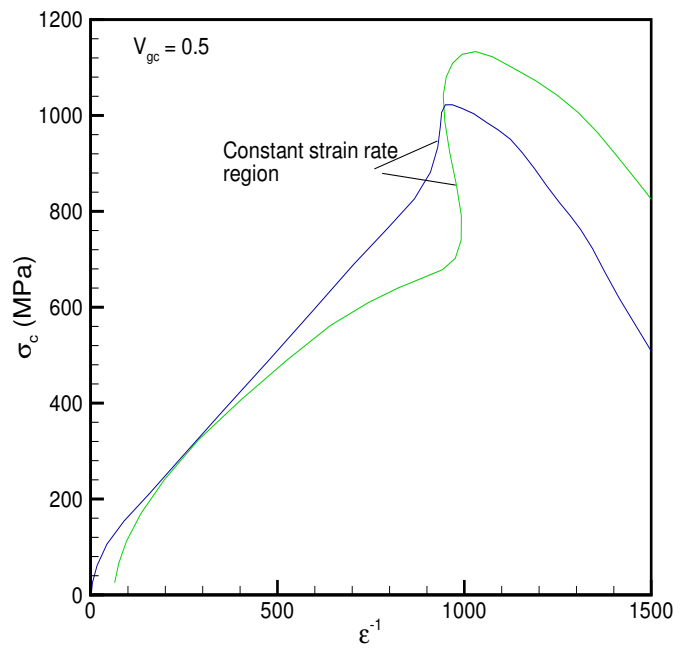

Figure 6: Variation of stress with strain rate for $V_{g c}=0.5$

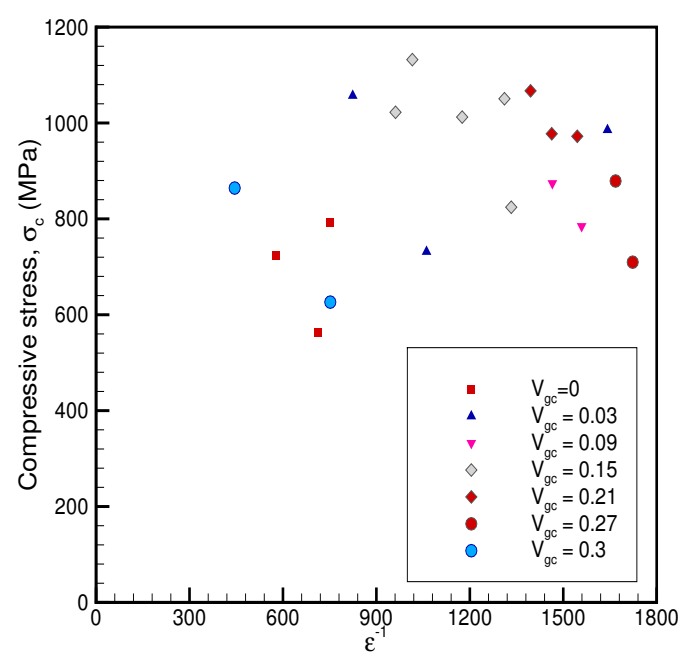

Figure 7: Variation of failure strength with strain rate, for different hybrid ratios

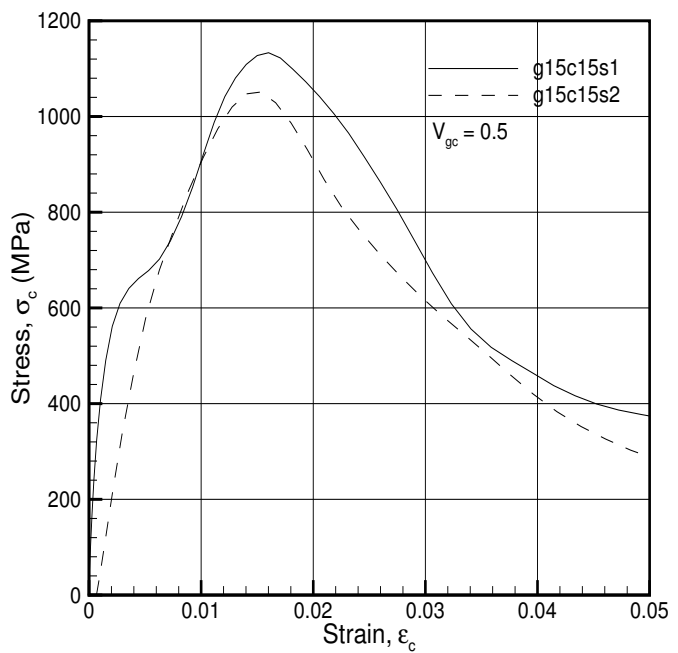

Figure 8: Typical stress strain curves for a composite of hybrid ratio, $V_{g c}=0.5$ 


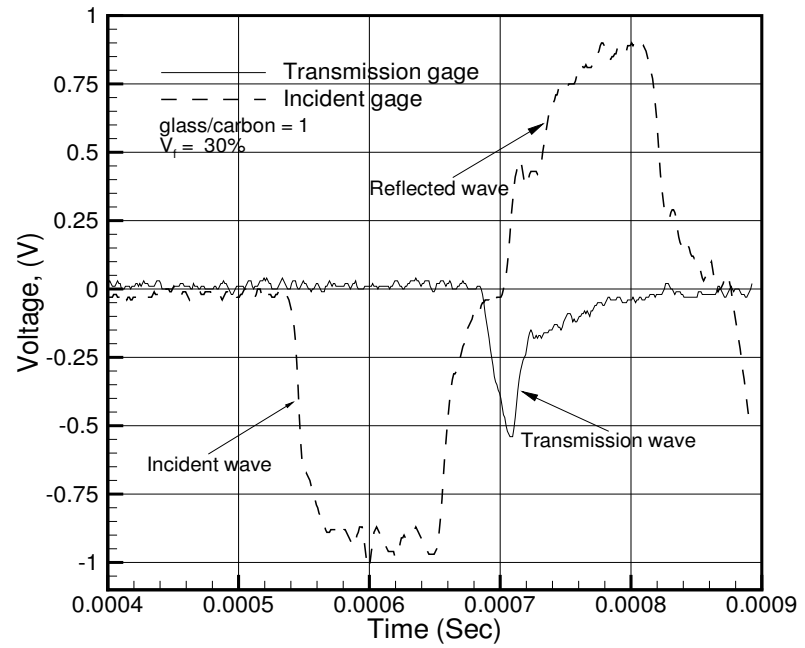

Figure 9: A typical strain gage signal obtained from the SHPB test on a hybrid composite of $\mathrm{g} / \mathrm{c}$ ratio 1

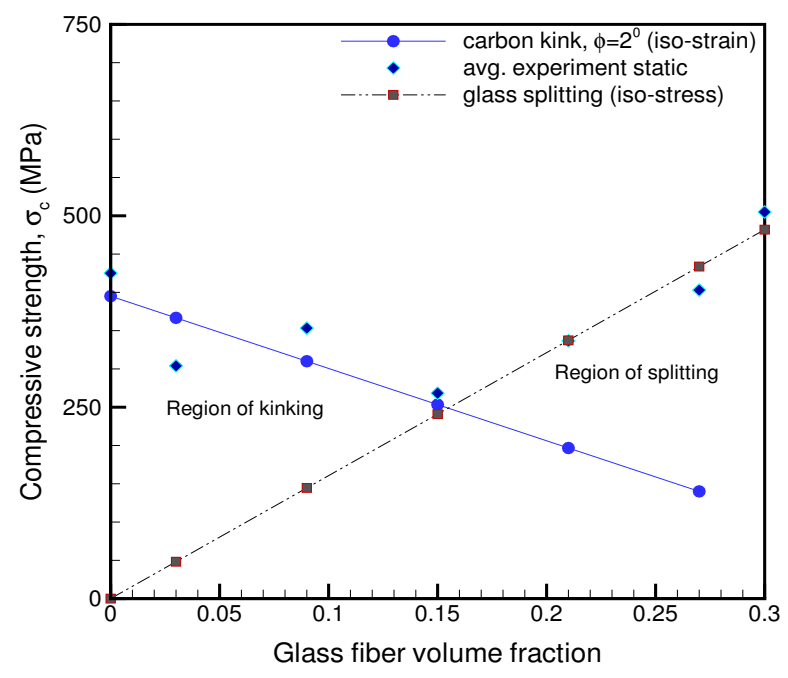

Figure 11: Failure model predictions for hybrid composites (static loading)

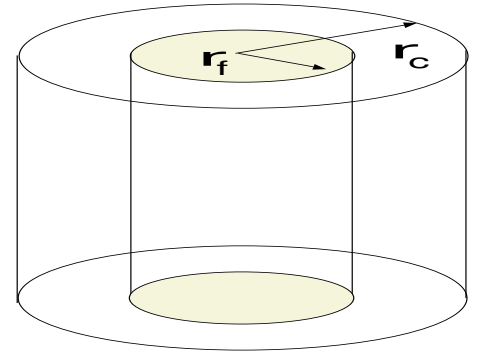

a) Conventional two cylinder concentric model RVE (inner fiber/outer radius)

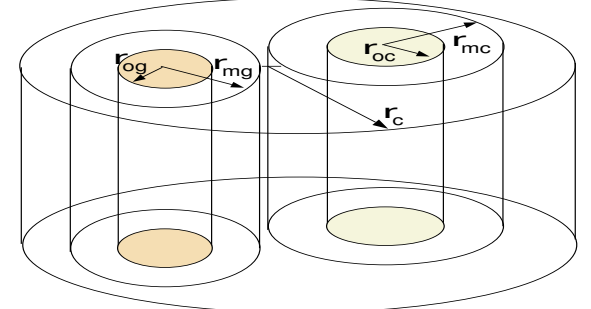

b) Modified to a Hybrid composite case with outer cylinder assumed to consist of individual concentric cylinders of fiber/matrix

Figure 10: Concentric cylinder models

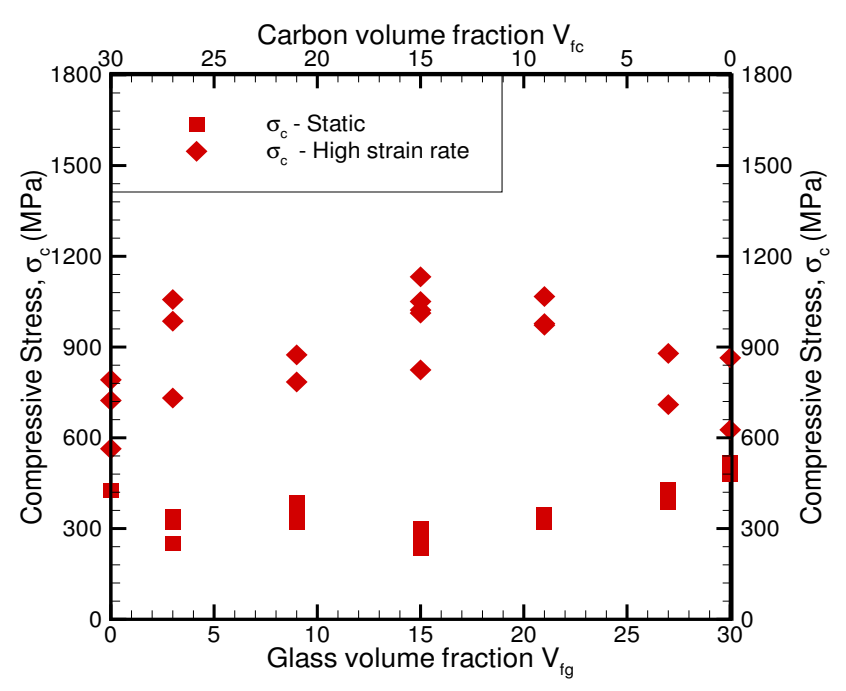

Figure 12: Variation of failure strength with strain rate, for different hybrid ratios 